Nikos I. Karachalios*, Paris Kyriazopoulos and Konstantinos Vetas

\title{
Excitation of Peregrine-Type Waveforms from Vanishing Initial Conditions in the Presence of Periodic Forcing
}

https://doi.org/10.1515/zna-2018-0540

Received December 6, 2018; accepted February 7, 2019; previously published online March 9, 2019

\begin{abstract}
We show by direct numerical simulations that spatiotemporally localised waveforms, strongly reminiscent of the Peregrine rogue wave, can be excited by vanishing initial conditions for the periodically driven nonlinear Schrödinger equation. The emergence of the Peregrinetype waveforms can be potentially justified, in terms of the existence and modulational instability of spatially homogeneous solutions of the model and the continuous dependence of the localised initial data for small time intervals. We also comment on the persistence of the above dynamics, under the presence of small damping effects, and justify that this behaviour should be considered as far from approximations of the corresponding integrable limit.
\end{abstract}

Keywords: Forced Nonlinear Schrödinger Equation; Modulational Instability; Peregrine Soliton; Time-Periodic Driver; Vanishing Initial Conditions.

\section{Introduction}

A crucial and intriguing question in nonlinear dynamics concerns the persistence of dynamical features of integrable systems in the presence of perturbations. In the context of the nonlinear Schrödinger (NLS) partial differential equations, and particularly for the integrable NLS (with a focusing, cubic nonlinearity), one of these features that is receiving tremendous interest concerns the emergence of rogue waves: extreme wave events possessing spatiotemporal localisation and large amplitude, which are mathematically described by its class of rational solutions. For the fundamental representatives of this class, namely, the Peregrine rogue wave (PRW), and the space- or

\footnotetext{
*Corresponding author: Nikos I. Karachalios, Department of Mathematics, University of the Aegean, Karlovassi, GR 83200 Samos, Greece, E-mail: karan@aegean.gr. https://orcid.org/0000-00025580-3957

Paris Kyriazopoulos and Konstantinos Vetas: Department of Mathematics, University of the Aegean, Karlovassi, GR 83200 Samos, Greece
}

time-periodic solutions as the Akhmediev and Kuznetsov$\mathrm{Ma}(\mathrm{KMb})$ breathers, respectively [1-4], their physical relevance is justified by numerous episodes in the ocean [5-7] and experimental observations in hydrodynamics [8-10], nonlinear optics and lasers [11-13], superfluidity [14], and plasma physics [15]. Such natural and experimental evidences motivated recent advances on the predictability of extreme wave events based on studies analysing the interactions of energy localisation and strong local nonlinearity $[16,17]$.

In the context of the aforementioned persistence question, the robustness of rational solutions and rogue wave dynamics in the presence of perturbations has been identified for various special cases of extended NLS equations. Representative key works refer to third-order (including modified Hirota [18] and Dysthe [19] equations) [20-22] as well as fourth- [23, 24] and fifth-order [25] models. Important extensions to coupled equations and systems include [26] for parity-time symmetric systems, [27] for NLS systems with derivative nonlinearities, and [28] for Manakov systems (physically relevant in the context of BoseEinstein condensates). In a different perspective, results on the spectral analysis of the PRW as a limiting case of the $\mathrm{KMb}$ are presented in [29]. Furthermore, the findings of [30] suggest that a suitably defined dispersion or nonlinearity management, if applied to a continuous wave (cw) background, may effectively stabilise the supported PRW and $\mathrm{KMb}$ waveforms.

In this spirit, and motivated by key works on the linearly forced/driven NLS equations in the context of rogue waves and the robustness of localised waveforms in nearly integrable systems [31-34], we consider, instead of the linear forcing, the action of an external, time-periodic driver. The relevance of such a driving term, arising in a number of physical systems (as in the theory of charge-density waves and plasma physics), has been extensively analysed in [34]. Then, continuing along the lines of our recent work [35], we examine, starting herein numerically, the potential emergence of spatiotemporal algebraically decaying waveforms in the dynamics of the associated, periodically driven NLS model.

However, the approach we will follow regarding the initial conditions differs drastically from investigations 
exciting PRWs, from initial data defined as interactions between a pulse of small amplitude and a cw [35-38]. Instead of such initial conditions, we ask for the possibility to excite PRWs from vanishing initial conditions, decaying either at an algebraic or exponential rate.

The answer is positive: the main finding is that extreme waveforms, strongly reminiscent to the PRW, can be excited from the decaying initial data. Their profile and statiotemporal decay rates are close to that of the analytical PRW solution. An important feature of this finding is that these PRW-solitonic structures emerge on the top of a finite background, which is formed at the early stages of the evolution, although the initial condition decays to zero. The birth of the PRW-type waveforms can be potentially understood by a synergy of the modulational instability (MI) of the cw solutions of the model and the persistence of the localisation of the initial condition on the top of the emerged unstable background. In other words, for vanishing initial data, in the presence of the periodic forcing, the system self-induces the effects of the MI mechanism analysed in [35-38], for the excitation of PRWs.

The article is structured as follows: In Section 2, we report the results of the numerical simulations and analytical considerations on the stability of spatial homogeneous solutions. We also briefly comment on the dynamics of the linearly damped and forced model, and of the integrable NLS, initiated from the same, decaying initial conditions. In Section 3, we summarise our results with an eye towards future work.

\section{Numerical Results}

\subsection{Brief Description of the Model}

In this section, we report the results of direct numerical simulations on the dynamics of the periodically driven, NLS equation

$$
\begin{aligned}
& \mathrm{i} u_{t}+\frac{v}{2} u_{x x}+\sigma|u|^{2} u=\Gamma \exp (\mathrm{i} \Omega t), \quad v>0, \sigma>0, \\
& \Gamma \in \mathbb{C}, \quad \Omega \in \mathbb{R} .
\end{aligned}
$$

In (1), the parameter $v$ is the second-order (group velocity) dispersion, and $\sigma$ is the strength of the nonlinearity. The parameters $\Gamma$ and $\Omega$ correspond to the amplitude and frequency of the driver, respectively. Let us recall that under the change of variable $u \rightarrow u \exp (\mathrm{i} \Omega t)(1)$ is transformed to the autonomous equation $\mathrm{i} u_{t}+\frac{v}{2} u_{x x}+\left(\sigma|u|^{2}-\right.$
$\Omega) u=\Gamma$. In what follows, we shall restrict to the case $\Gamma \geq 0 .{ }^{1}$

Equation (1) defines a nonintegrable perturbation of the focusing integrable NLS, which corresponds to the case $\Gamma=0$. It is one of the fundamental partial differential equations exhibiting complex [39], even spatiotemporal chaotic behaviour [40, 41], together with its dissipative counterparts [42-44]. The impact of the breaking of the hyperbolic structure of the integrable NLS, in the emergence of complex dynamics for the damped and forced models, has been rigorously analysed in [45-48]. For equations incorporating higher-order dissipation, we refer to $[34,49]$ and references therein.

The numerical experiments will simulate the dynamics of (1), excited by either the quadratically decaying initial condition

$$
u_{0}(x)=\frac{1}{1+x^{2}},
$$

or the exponentially decaying

$$
u_{0}(x)=\alpha \operatorname{sech}(\beta x) \text {, for some } \alpha, \beta>0 \text {, }
$$

which resembles the profile of a bright-soliton solution of the integrable NLS. The model will be supplemented with periodic boundary conditions

$$
u(x+2 L, t)=u(x, t), \text { for all } t \geq 0 .
$$

\subsection{Background}

The system was integrated by using a pseudospectral method for the spatial discretisation and an adaptive step Runge-Kutta method for the time stepping. Concerning the implementation of the periodic boundary conditions, for cases (2) and (3) of the initial data, let us recall the following: These conditions are strictly satisfied only asymptotically, as $L \rightarrow \infty$. For a finite length $L$, the initial profiles, as well as their spatial derivatives, have jumps across the end points of the interval $[-L, L]$. However, these jumps have negligible effects in the observed dynamics, as they are either of order $1 /\left(1+L^{2}\right)$ or $\exp (-L)$. More precisely, as the smallest value for $L$ used herein is $L=100$, the effects are of order $\mathcal{O}\left(10^{-4}\right)$ or less. The characterisation of the numerically observed spatiotemporal

1 For the generic case of $\Gamma \in \mathbb{C}$, we remark that amplitude and phase may seriously affect the dynamics that will be discussed herein. Relevant investigations are in progress and will be considered elsewhere. 
localised waveforms as extreme is made by a comparison [50] against time translations of the analytical PRW solution of the integrable NLS:

$$
u_{\mathrm{PS}}\left(x, t ; P_{0}\right)=\sqrt{P_{0}}\left[1-\frac{4\left(1+\frac{2 \mathrm{i} t}{\Lambda}\right)}{1+\frac{4 x^{2}}{K_{0}^{2}}+\frac{4 t^{2}}{\Lambda^{2}}}\right] \exp \left(\frac{\mathrm{it}}{\Lambda}\right),
$$

where the parameters $\Lambda=\frac{1}{\sigma P_{0}}$ and $K_{0}=\sqrt{\nu \Lambda}$. Note that the PRW solution decays algebraically both in time and space, on the top of the continuous background of power $P_{0}$. We denote these translations as $u_{\mathrm{PS}}\left(x, t-t^{*} ; P_{0}\right)$. The time $t^{*}$ and power $P_{0}$ are numerically detected, as described below.

\subsection{Results of the Direct Numerical Simulations}

Figure 1 shows snapshots of the evolution of the density $|u(x, t)|^{2}$ of the numerical solution of the problem (1), (2), (4), for $\nu=\sigma=1, \Gamma=0.5$, and $\Omega=1$, for the algebraically decaying initial condition (2). We observe that the initial datum evolves towards a localised waveform, which is strongly reminiscent of a PRW. The numerical solution is plotted by the continuous (blue) curve, against the dashed (red) curve depicting the evolution of the PRW profile (5), $u_{\mathrm{PS}}(x, t-2.415 ; 0.84)$. The maximum amplitude of the event is attained at $t^{*}=2.415$. The time $t^{*}$ is used to define the time translation of the analytical PRW solution of the integrable NLS. The power of its background $P_{0}=0.84$ is numerically detected, so that the amplitude of the analytical PRW coincides with the maximum amplitude of the numerical event. Note that for the above set of parameters, we found that $K_{0}=1.54$ and $\Lambda=1.19$.
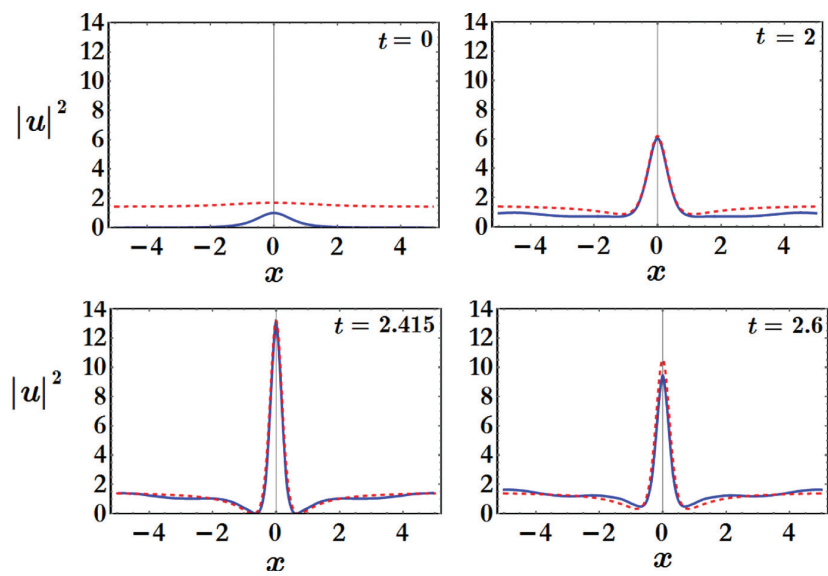

For $t \in[1.3,2.5]$, the centred localised waveform exhibits an algebraic in time growth/decay rate, close to that of the PRW soliton; notably, both the time-growing and then time-decaying centred profiles appear to manifest a locking to a PRW-type mode, as it is depicted in the left panel (a) of the top row of Figure 2, showing the time evolution of the density of the centre $|u(0, t)|^{2}$, for $t \in[0,3]$. The middle panel (b) of the top row of Figure 2 shows a detail of the spatial profile of the maximum event at $t^{*}=2.415$, close to the right of the two symmetric minima of the exact PRW $u_{\mathrm{PS}}(x, t-2.415 ; 0.84)$. This detail illustrates that the emerged extreme event preserves the algebraic spatial decay of the PRW soliton.

The top right panel (c) of Figure 2 depicts a rescaled, extended view of the maximum extreme event, plotted for $x \in[-L, L]$. It reveals a remarkable feature of the dynamics: the extreme event occurs on the top of a finite background of amplitude $|h|^{2} \sim 1.19$, which is formed at the early stages of the evolution of the vanishing initial condition; on the one hand, we observed that the core of the PRW-solitonic structure is proximal to the analytical PRW of the integrable NLS with $P_{0}=0.84$ (as numerically detected by the fitting argument of [50] discussed above), and on the other hand, it does not tend to the background of unit amplitude as the PRW of the integrable NLS when $v=\sigma=1$. This fact that the amplitude of the background of the PRW-type event differs from $P_{0}=0.84$ or $P_{0}=1$ suggests that it may be determined by the driver, as it will be analysed below. Note that the same effects were observed for increased values of $L$.

The bottom row of Figure 2 depicts contour plots of the spatiotemporal evolution of the density $|u(x, t)|^{2}$. The bottom left panel (d) portrays the evolution for $t \in[0,10]$. The PRW-type soliton discussed above is the

Figure 1: (Colour online) Snapshots of the evolution of the density $|u(x, t)|^{2}$ [solid (blue) curves], for the initial condition (2). Parameters: $\nu=1, \sigma=1, \Gamma=0.5, \Omega=1, L=100$. The density of the numerical solution of (1) is compared against the density of the PRW of the integrable NLS (5), $u_{\mathrm{PS}}(x, t-2.415 ; 0.84)$ [dashed (red) curves], with $K_{0}=1.54$ and $\Lambda=1.19$. 

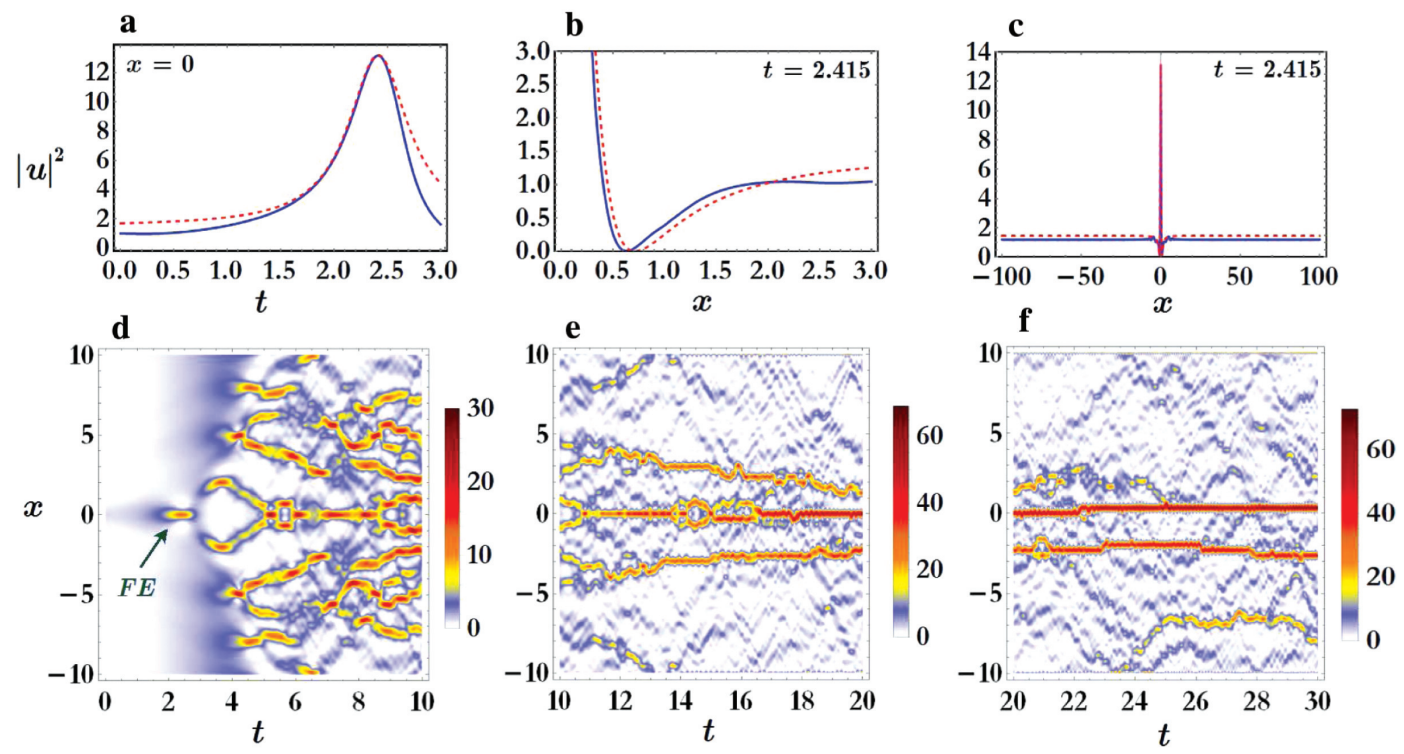

Figure 2: (Colour online) Parameters: $\nu=1, \sigma=1, \Gamma=0.5, \Omega=1, L=100$. Top left panel (a): evolution of the density of the centre, $|u(0, t)|^{2}$, for the initial condition (2), against the evolution of the density of the centre of the PRW (5), $u_{\mathrm{PS}}(x, t-2.415 ; 0.84)$, with $K_{0}=1.54$ and $\Lambda=1.19$. Top middle panel (b): a detail of the spatial profile of the maximum event at $t^{*}=2.415$, close to the right of the two symmetric minima of the exact PRW $u_{\mathrm{PS}}(x, t-2.415 ; 0.84)$. Top right panel (c): Another view of the numerical density, at time $t^{*}=2.415$, where the extreme event attains its maximum amplitude, for $x \in[-100,100]$. The PRW-type structure is formed on a finite background. Bottom panels: Contour plots of the spatiotemporal evolution of the density, for the above evolution, for $t \in[0,10](\mathrm{d})$, for $t \in[10,20](\mathrm{e})$, and $t \in[20,30](\mathrm{f})$.

first extreme event (FE) corresponding to the spot marked by the arrow. After its formation, the sustaining finite background exhibits MI dynamics, characterised by the emergence of large-amplitude localised modes. A first interesting effect is that the solution preserves at the early stage of the evolution the even spatial symmetry $u(x, t)=$ $u(-x, t)$. The even symmetry breaking due to the presence of the driver occurs for $t \gtrsim 14$, as shown in the contour plot of the bottom middle panel (e), which portrays the dynamics for $t \in[10,20]$. A second interesting effect is that the later stages of MI are manifested by spatial energy localisation (initiated prior to the even symmetry breaking) and the formation of extreme amplitude solitary modes. The survived solitary modes are dominating in the dynamics, as shown in the right contour plot (f) (which shows the relevant evolution for $t \in[20,30])$, and their amplitude is increasing.

Figure 3 is an attempt to shed light to the structure of the above solitary modes. The left panel (a) depicts the evolution of the density of the centre for $t \in[0,16]$, showing that it undergoes chaotic oscillations. The oscillations in the subinterval $[10,16]$ correspond to those of the top of the solitary mode, which was depicted in the contour plot of the evolution of the density of Figure 2e. This is first evidence that the solitary mode possesses the structure of a (large amplitude) "chaotic soliton" in the sense of $[42-44,51]$ than a breather. A second evidence is illustrated in the middle panel (b), showing a plot of the evolution of the density of $x=0.33$, i.e. $|u(0.33, t)|^{2}$, for $t \in[28,30]$; it is actually a detail - in this time subinterval - of the evolution of the solitary mode depicted in the contour plot of Figure 2e. The centred mode has slightly slided at $x=0.33$, and the oscillations of the mode are reminiscent of those presented in [42, Fig. 1, p. 4]. We may conjecture that the system, for the considered example of parameters, is locked to a "chaotic" soliton and not to a large-amplitude breathing mode. Nevertheless, varying the parameters of the driver, the appearance of more breather-like waveforms may not be excluded (at least, at the early stages of the evolution), as shown in Figure 4, depicting the dynamics when $\Gamma=1$ and $\Omega=2.7$. These breather-like modes may evolve to the aforementioned "chaotic" solitons at later stages of the dynamics.

The numerical results that follow come out from an indicative study on the dependencies of the above dynamics on the parameters of the driver. In Figures 5 and 6, we fixed $\Gamma=0.5$ and $\nu=\sigma=1$ as above, and we varied its frequency $\Omega$. The left panel (a) of Figure 5 depicts the contour plot of the spatiotemporal evolution of the density for $\Omega=0.5$ and the right panel (b) for $\Omega=1.5$. Despite some changes in the patterns, the overall picture of the dynamics observed in the case $\Omega=1$ (large-amplitude 

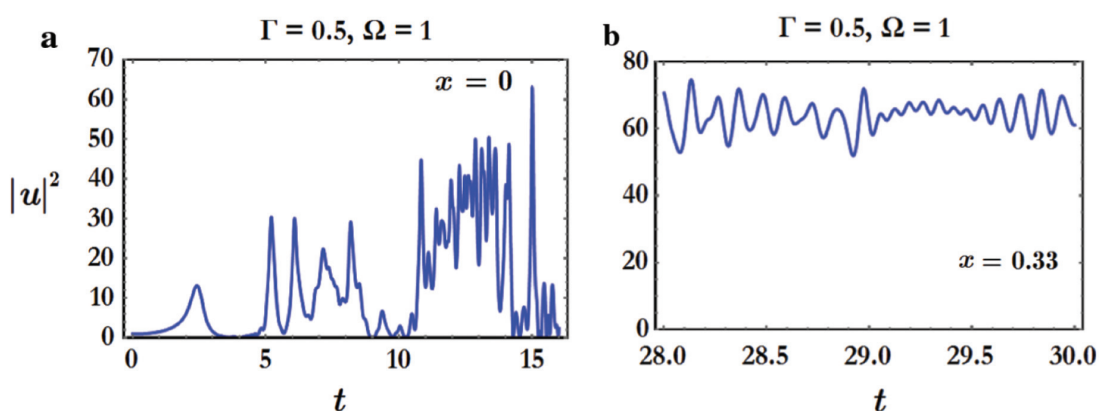

Figure 3: Parameters: $v=1, \sigma=1, \Gamma=0.5, \Omega=1, L=100$. Left panel (a): Temporal evolution of the density of the centre $|u(0, t)|^{2}$, for the initial condition (2). Middle panel (b): A detail of the temporal evolution of the density at $x=0.33\left(|u(0.33, t)|^{2}\right)$, for $t \in[28,30]$.
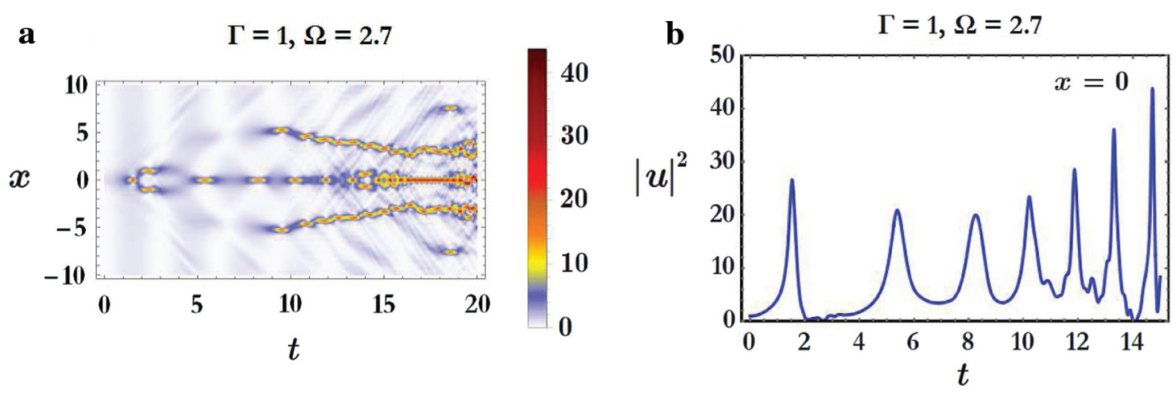

Figure 4: Parameters: $\nu=1, \sigma=1, \Gamma=1, \Omega=2.7, L=100$, and initial condition (2). Left panel (a): Contour plot of the spatiotemporal evolution of the density for $t \in[0,20]$. Right panel (b): Temporal evolution of the density of the centre $|u(0, t)|^{2}$, for $t \in[0,14]$.
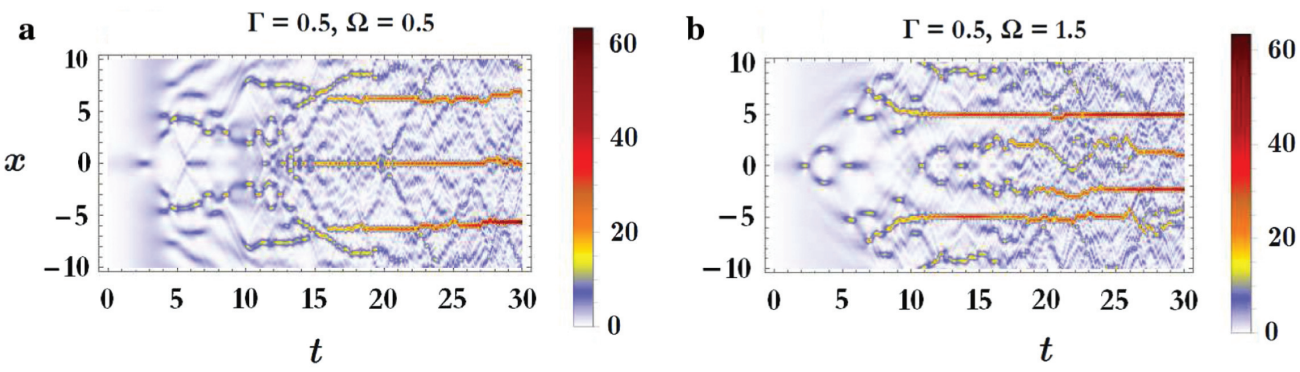

Figure 5: (Colour online) Parameters: $\nu=1, \sigma=1, \Gamma=0.5, L=100$, and initial condition (2). Left panel (a): Contour plot of the spatiotemporal evolution of the density for $t \in[0,30]$, when $\Omega=0.5$. Left panel (b): as in panel (a), but for $\Omega=1.5$.

solitons, following after the emergence of extreme FE) persists for both examples of $\Omega=0.5<1$ and $\Omega=1.5>1$, respectively.

Drastic changes appear for larger values of the driver's frequency $\Omega$. These changes are illustrated in columns (a) to (c) of Figure 6. In each column, the upper panel shows the temporal evolution of the density of the centre $|u(0, t)|^{2}$ for $t \in[0,30]$, and the bottom panel shows a contour plot of the spatiotemporal evolution of the density for $x \in[-10,10]$ and $t \in[0,30]$. Column (a) depicts the numerical results for $\Omega=2$, a value that in the present study - for the considered set of parameters can be viewed as "critical": The large-amplitude peak of the density of the centre observed in the top panel (a) corresponds to a PRW-type event - the spot of the bottom panel (a). Remarkably, afterwards - in contrast with the previous observations - we see that the large-amplitude, "chaotic solitary" modes disappear; the later stages of the dynamics are manifested by small amplitude chaotic oscillations instead, as depicted in the inset of the top panel (a).

Increasing the driver's frequency to $\Omega=4$, we observe in column (b) yet another remarkable effect: the disappearance of the PRW-type events. The dynamics are locked to a spatially localised mode whose top is oscillating in time almost periodically with moderate amplitudes. The frequency of the oscillations of the top of such "quasiperiodic" solitary modes seems to be dictated by 

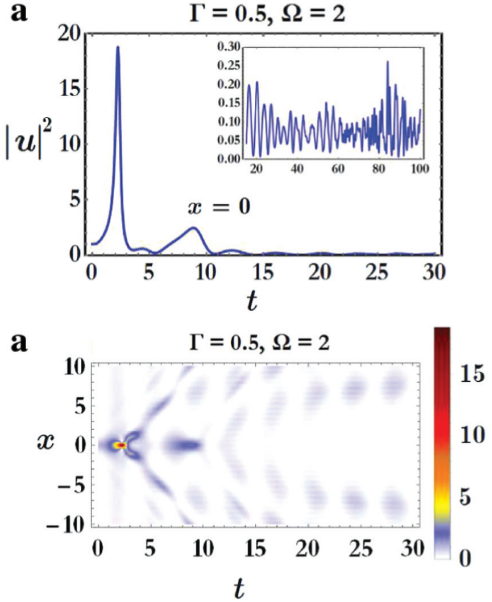
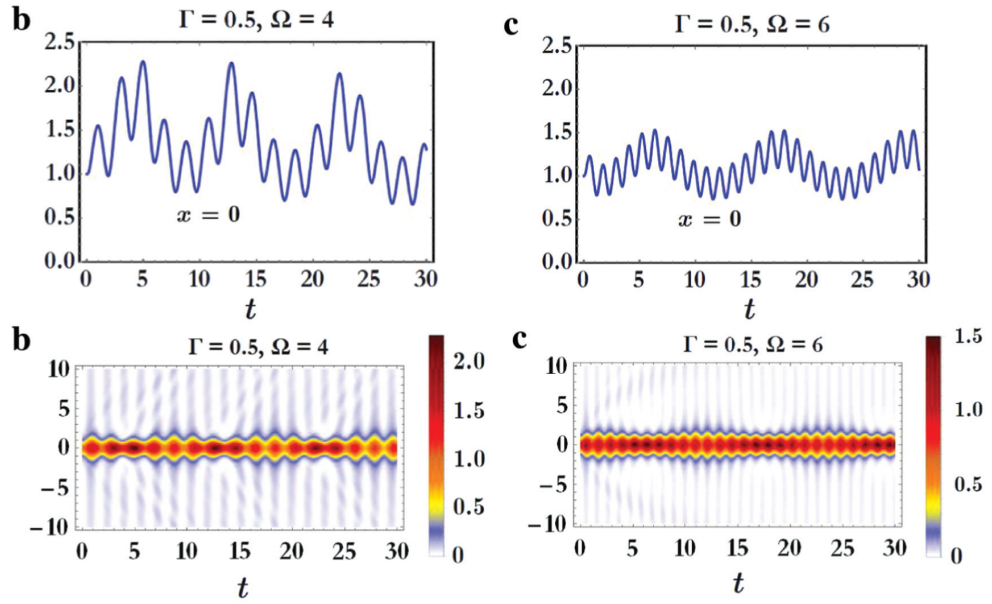

Figure 6: (Colour online) Parameters: $v=1, \sigma=1, \Gamma=0.5, L=100$, and initial condition (2). Column (a): The upper panel (a) shows the temporal evolution of the density of the centre $|u(0, t)|^{2}$, for $t \in[0,30]$, when $\Omega=2$. The bottom panel (a) shows the contour plot of the spatiotemporal evolution of the density for $x \in[-10,10]$ and $t \in[0,30]$, when $\Omega=2$. Column (b): Same as in column (a), but for $\Omega=4$. Column (c). Same as in column (a), but for $\Omega=6$.

the frequency of the driver and increasing, as shown in column (c), depicting the relevant evolution for the increased value of $\Omega=6$. When $\Omega$ is further increased, the frequency of the above oscillations is also increasing, suggesting that the dynamics tend to lock to a stationary soliton. This is expected, since in the limit of large $\Omega$, as the period of the oscillations is dictated by the frequency of the driver, it should tend to zero.

Next, keeping the driver's frequency fixed to $\Omega=1$, a similar dynamical phenomenology to the one presented in Figure 2 emerged for the reduced forcing amplitude $\Gamma=0.25$. The dynamics for this example are summarised in Figure 7. In this case, the FE is found to be close to the PRW-soliton $u_{\mathrm{PS}}(x, t-3.33 ; 1.06)$ of the integrable limit, with $K_{0}=0.97$ and $\Lambda=0.94$. The presentation is the same as in Figure 2. The top left panel (a) shows the time evolution of the density of the centre, where its time growth and time decay is still close to the PRW for $t \in[2,3.5]$. The top middle panel (b) illustrates that the numerical solution, when the FE attains its maximum density at $t^{*}=3.33$, yet captures the profile of the PRW around its symmetric minima, even closer than the case of $\Gamma=0.5$. The whole profile of the $\mathrm{FE}$ at the time of its maximum density is depicted in the top right panel (c). As a result of the decreased forcing amplitude, the amplitude of the finite background supporting the PRW event is also decreased, i.e. $|h|^{2} \sim 0.34$. Accordingly, the emerging localised modes possess reduced amplitude. Additionally, we observe a delay in the emergence of the extreme amplitude solitary modes, as shown in the panels (d) to (f) of the bottom row, portraying contour plots of the spatiotemporal evolution of the density, for $t \in[0,60]$.
Proceeding to a progressive decrease of $\Gamma$, we observe a suppression of the extreme wave dynamics (similar to the case of increasing $\Omega$ ). These suppression effects are illustrated in Figure 8, where the presentation follows that of Figure 6: for $\Gamma=0.1$, we observe in column (a) that first the large-amplitude solitary structures disappear while the emergence of rogue waves still persists. This feature is shown by the large-amplitude peaks of the density of the centre shown in the top panel (a), which correspond to the localised spots of the contour plot portrayed in the bottom panel (a). Further suppression occurs for $\Gamma=0.05$ as shown in column (b) (manifested by the decrease of amplitude of the FE), while for $\Gamma=0.01$, the dynamics seems again to tend to lock to a stationary soliton.

A complete study of the bifurcations in the full parameter $(\Gamma, \Omega)$-parametric space, apart from being essential, might be a formidable task (as it may involve the nontrivial analysis of resonances given in [39-41]) and is beyond the scope of the present work. However, we may already conjecture on the dependencies of the exhibited dynamics on the amplitude $\Gamma$ and the frequency $\Omega$ of the driver. For instance, for fixed $\nu, \sigma, L$, we may identify thresholds $\Gamma_{\text {thresh }}$ and $\Omega_{\text {thresh }}$ such that for suitably fixed $\Omega$ (or $\Gamma$ ), if $\Gamma>\Gamma_{\text {thresh }}$ (or $\Omega<\Omega_{\text {thresh }}$ ), extreme wave dynamics emerge. The above numerical studies provided the following examples for the thresholds: we found that for $\nu=\sigma=$ 1 and $L=100$, when $\Omega=1, \Gamma_{\text {thresh }}<0.05$, and when $\Gamma=0.5$, then $\Omega_{\text {thresh }}>2$. Furthermore, in the suppression regimes, in the limit of small $\Gamma$ (for fixed $\Omega$ ) or in the limit of large $\Omega$ (for fixed $\Gamma$ ), the dynamics tend to lock to a stationary soliton of the integrable NLS. This is expected, as in the limit of small $\Gamma$, the system approximates the integrable limit. 

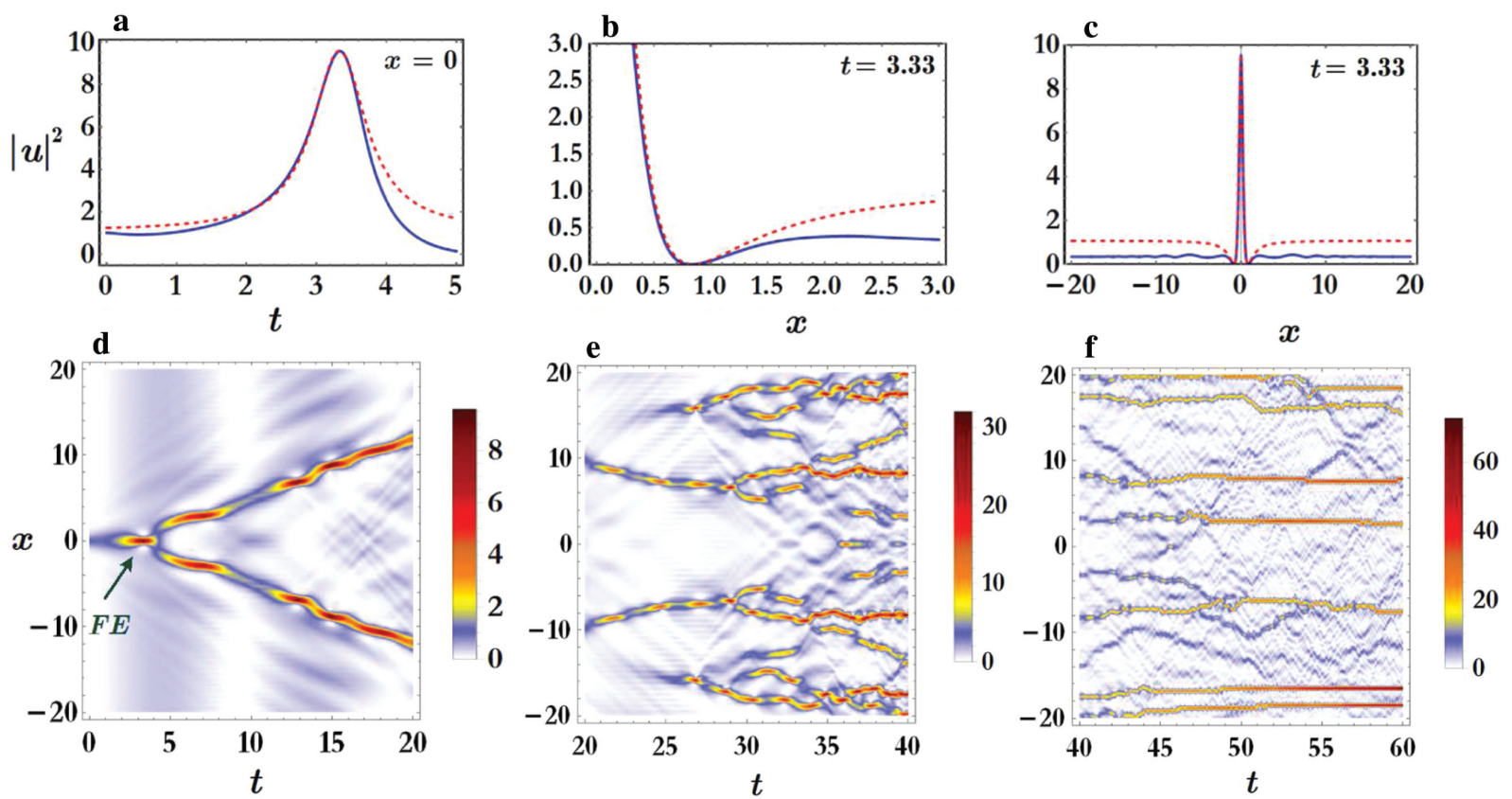

Figure 7: (Colour Online) Dynamics of the initial condition (2), for forcing amplitude $\Gamma=0.25$ and the rest of parameters fixed as in Fig. 2. (a) Evolution of the density of the center, $|u(0, t)|^{2}$, for the initial condition (2), against the evolution of the density of the center of the PRW (5), $u_{\mathrm{PS}}(x, t-3.33 ; 1.06)$, with $K_{0}=0.97$ and $\Lambda=0.94$. (b) A detail of the spatial profile of the maximum event at $t^{*}=3.33$, close to the right of the two symmetric minima of the exact PRW $u_{\mathrm{PS}}(x, t-3.33 ; 1.06)$. (c) Another view of the numerical density, at time $t^{*}=3.33$, where the extreme event attains its maximum amplitude, for $x \in[-20,20]$. The PRW-type structure is still formed on a finite background. Bottom panels: Contour plots of the spatiotemporal evolution of the density, for the above evolution, for $t \in[0,20](\mathrm{d})$, for $t \in[20,40](\mathrm{e})$, and $t \in[40,60]$ (f).
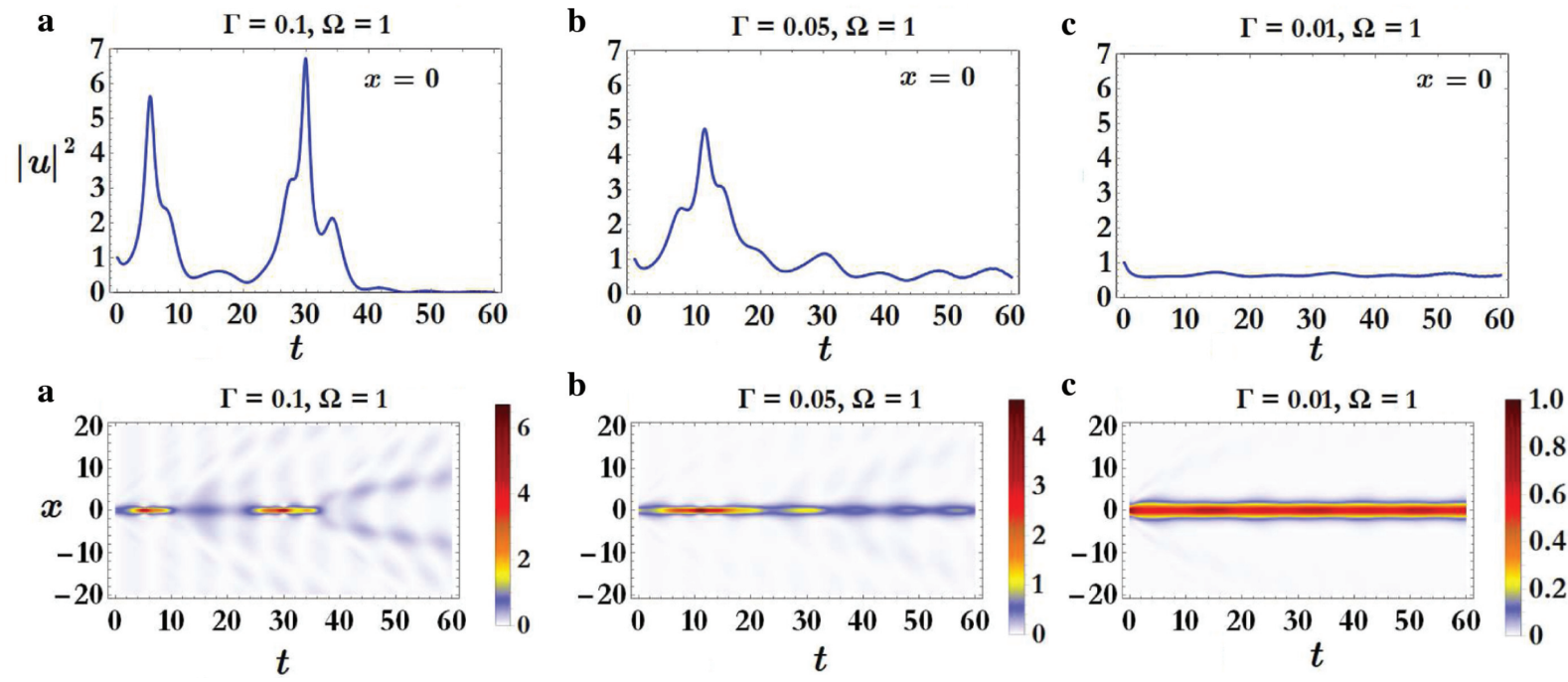

Figure 8: (Colour online) Parameters: $\nu=1, \sigma=1, \Omega=1, L=100$, and initial condition (2). Column (a): The upper panel (a) shows the temporal evolution of the density of the centre $|u(0, t)|^{2}$, for $t \in[0,30]$, when $\Gamma=0.1$. The bottom panel (a) shows the contour plot of the spatiotemporal evolution of the density for $x \in[-10,10]$ and $t \in[0,30]$, when $\Gamma=0.1$. Column (b): Same as in column (a), but for $\Gamma=0.05$. Column (c): Same as in column (a), but for $\Gamma=0.01$.

The above observations will be further underlined by the comments on the behaviour of the integrable limit $\Gamma=0$, for the same type of vanishing conditions.

\subsection{Effects from Continuous Wave Solutions}

The existence and stability properties of cw solutions of the forced NLS (1) should have an important role on the 
birth of the transient PRW-type dynamics. In what follows, we fix for simplicity $\nu=\sigma=1$ in (1), corresponding to the presented results of the numerical simulations discussed above. We consider spatially homogeneous solutions, of the form

$$
u(x, t)=h e^{\mathrm{i} \Omega t}, \quad h \in \mathbb{C} .
$$

There exist for (1), under the dispersion relation

$$
|h|^{2} h=\Gamma+h \Omega .
$$

For $\Gamma>0$, the case we are restricted herein, (7) has only real solutions for $h$ : Indeed, let $h=A+\mathrm{i} B$. Then, its substitution to (7) is leading to the following equations for $A$ and $B$ :

$$
\begin{aligned}
& A\left(A^{2}+B^{2}\right)-\Omega A=\Gamma, \\
& B\left(A^{2}+B^{2}\right)-\Omega B=0 .
\end{aligned}
$$

From the second equation, we have that either $B=0$, or $|h|^{2}=A^{2}+B^{2}=\Omega$. Inserting the latter to the first equation for $A$, we get that $\Gamma=0$. Thus, for $\Gamma>0$ (and generically, for $\Gamma \in \mathbb{R}$ ), solutions (6) exist only with $h \in \mathbb{R}$. Now, consider a perturbation to the solution (6), of the form

$$
u(x, t)=\left[h+\varepsilon u_{1}(x, t)\right] e^{\mathrm{i} \Omega t},
$$

for small $\varepsilon>0$, which we insert into (1). By using the dispersion relation (7) and linearising the system, i.e. neglecting terms of order $\varepsilon^{2}$ and higher, we derive the equation this small influence satisfies:

$$
\mathrm{i} \partial_{t} u_{1}+\frac{1}{2} \partial_{x}^{2} u_{1}+2 h^{2} u_{1}+h^{2} \overline{u_{1}}-\Omega u_{1}=0 .
$$

To examine MI, we may assume that the perturbation $u_{1}$ is harmonic, i.e.

$$
u_{1}(x, t)=c_{1} e^{i(k x-\omega t)}+c_{2} e^{-i(k x-\omega t)},
$$

where $k$ and $\omega$ denote the wavenumber and the frequency of the perturbation, respectively. Next, substitution of the expression (10) for $u_{1}$ in the linearised (9) yields the following algebraic system for $c_{1}$ and $c_{2}$ :

$$
\begin{aligned}
& \left(-\frac{k^{2}}{2}+\omega-\Omega+2 h^{2}\right) c_{1}+h^{2} c_{2}=0, \\
& h^{2} c_{1}+\left(-\frac{k^{2}}{2}-\omega-\Omega+2 h^{2}\right) c_{2}=0 .
\end{aligned}
$$

Seeking for nontrivial solutions $c_{1}$ and $c_{2}$ of the above system, we require the relevant determinant to be zero; this way, we obtain the following dispersion relation:

$$
\omega^{2}=\frac{k^{4}}{4}+k^{2}\left(\Omega-2 h^{2}\right)+\left(3 h^{4}-4 \Omega h^{2}+\Omega^{2}\right) .
$$

The perturbation (8) suggests that solutions (6) are modulationally unstable if $\omega$ is complex, i.e. when the right-hand side of (11) is negative. Solving the equation

$$
k^{4}+k^{2}\left(4 \Omega-8 h^{2}\right)+12 h^{4}-16 \Omega h^{2}+4 \Omega^{2}=0,
$$

in terms of $k$, we find the following solutions:

$$
k_{1,2}= \pm \sqrt{2} \sqrt{h^{2}-\Omega}, \quad k_{3,4}= \pm \sqrt{2} \sqrt{3 h^{2}-\Omega} .
$$

The roots (12) define the instability bands of the cw solutions (6). There is not a loss of generality to be restricted for $k>0$. Then, the instability bands are defined as follows:

1. $I_{a}=\left[0, k_{3}\right]=\left[0, \sqrt{2} \sqrt{3 h^{2}-\Omega}\right]$, if $\frac{\Omega}{3}<h^{2}<\Omega$.

2. $I_{b}=\left[k_{1}, k_{3}\right]=\left[\sqrt{2} \sqrt{h^{2}-\Omega}, \sqrt{2} \sqrt{3 h^{2}-\Omega}\right]$,

$$
h^{2}>\Omega \text {. }
$$

Modulational instability has been proved an essential mechanism for the emergence of rogue waves [31-33]. In the light of the MI analysis recalled above, let us reconsider the dynamics presented in the example of Figures 1 and 2 . When $\Omega=1$ and $\Gamma=0.5$, (7) has one real root $h_{1}=1.19$. Thus, the corresponding cw solution (6), with $h=h_{1}$, exhibits its MI for wave numbers $k \in I_{b}$, as $h_{1}^{2}>\Omega$.

In this regard, we may conjecture that the background sustaining the PRW-type waveform shown in the snapshots of Figure 1 (and Fig. 2c) is self-induced as the system tends transiently to lock its cw solution in the presence of the forcing, e.g. the solution of amplitude $\left|h_{1}\right|^{2}$ in the considered example. However, this solution is modulationally unstable. Then, as a transient "metastability effect," the emergence of the PRW-type waveform is a result of the synergy of the preservation of the spatial localisation of the initial condition (due to continuous dependence on the initial data [35]) and of the MI of the sustaining cw solution: at a fixed time (recall the snapshot at time $t=2$ in Fig. 1), a pulse on a finite background has formed, although the evolution initiated by vanishing initial conditions; the system has self-induced the universal effects of the MI mechanism analysed in [36-38], which may lead to the birth of a PRW-type mode. The same mechanism may explain the dynamics portrayed in Figure 7.

We should remark that the above arguments are further supported by the detailed analysis of [39], on the adiabatic excitation and control of $N$-band solutions $(N$-phase waves) for the forced NLS. Particularly relevant is the analysis on the excitation of the spatially homogeneous (0band) solutions (6) from zero initial conditions, which are continuously synchronised with the driver (despite the variation of the driver's frequency); in our case, the vanishing initial conditions define perturbations of the zero 
background (being modulationally stable in the case of the integrable limit $\Gamma=0$ ).

\subsection{Comment on the Dynamics of the Damped Counterpart}

The effects of linear loss, solely influencing the evolution of Peregrine solitons in the 1D-focusing NLS, have been analysed via nonlinear spectral analysis in [52]; the unforced, damped NLS equation is physically significant in hydrodynamics and nonlinear optics $[32,33,53]$. Numerical and experimental studies [54] confirmed the observation of higher-order MI dynamics in water waves.

Here, we illustrate that dynamical behaviour of (1) discussed in the previous paragraphs seems to be robust for small damping strengths in the presence of the periodic forcing. For instance, this robustness was identified for the linearly damped counterpart of (1):

$$
\mathrm{i} u_{t}+\frac{\nu}{2} u_{x x}+\sigma|u|^{2} u+\mathrm{i} \gamma u=\Gamma \exp (\mathrm{i} \Omega t), \quad \gamma>0 .
$$

Yet, this model is of particular interest in various physical contexts, as in plasma physics [34, 44] (governing the dynamics of a collisional plasma driven by an external $\mathrm{rf}$ field). The dynamics of (13) are captured by a finite dimensional global attractor. For its existence and analyticity properties, we refer to $[55,56]$.

Figure 9 depicts the results of the numerical study for $\nu=2, \sigma=1$, damping strength $\gamma=0.02$, and $\Gamma=0.5$. This time, we have used the sech-profiled initial condition (3), for $\alpha=\sqrt{2}$ and $\beta=1$ corresponding to an exact stationary pulse of the integrable NLS (see below). As before, the left panel (a) shows the time evolution of the density of the centre $|u(0, t)|^{2}$, the middle panel (b) shows the detail of the emerged PRW-type event around the right of its minima, and the right panel (c) shows the profile of the

PRW-type event when its maximum density is attained at time $t^{*}=1.88$. The comparison is against the analytical PRW solution (5) $u_{\mathrm{PS}}(x, t-1.88$; 0.83$)$ with $K_{0}=1.55$ and $\Lambda=1.20$. Again, the excitation of the PRW-type waveforms can be explained as above, in terms of the modulation instability of the cw solutions (6) of (13) [44]. Additionally, it would be interesting to examine a potential stabilisation of the solitary modes observed in Figure 2e and $f$, in the presence of small damping, when possibly driven by two frequencies, as proposed in [57].

\subsection{Comment on the Dynamics of the Integrable NLS Limit}

It is important to note that the dynamics exhibited by the integrable NLS assuming the initial condition (2) or (3) totally differs from those of the forced (and damped) counterpart discussed in the previous paragraphs, although well understood. Figure 10 summarises the results of the numerical study of (1), for $\Gamma=0$.

The left column (a) depicts numerical results for the evolution of the initial condition (2), when $\nu=\sigma=1$. The upper panel (a) shows a snapshot of the density of the numerical solution at time $t=90$ [(blue) continuous curve], against the density of a numerically detected stationary pulse $|\Phi|^{2} \approx 0.54 \operatorname{sech}^{2}(0.73 x$ ) (dashed red curve). Let us recall the standard formula of the general bright-soliton solution of the cubic integrable NLS:

$$
\begin{aligned}
u(x, t)= & \sqrt{2\left|\frac{\theta-\kappa^{2} v / 2}{\sigma}\right|} \mid \operatorname{sech}\left[\sqrt{\left|\frac{\theta-\kappa^{2} \nu / 2}{\nu / 2}\right|}\right. \\
& (x-2 \theta \kappa t)] \exp [\mathrm{i}(\kappa x-\theta t)] .
\end{aligned}
$$

When $\sigma=v=1$ and $\kappa=0$, the corresponding stationary solution has density $|u|^{2}=2|\theta| \operatorname{sech}^{2}(\sqrt{2|\theta|} x)$.
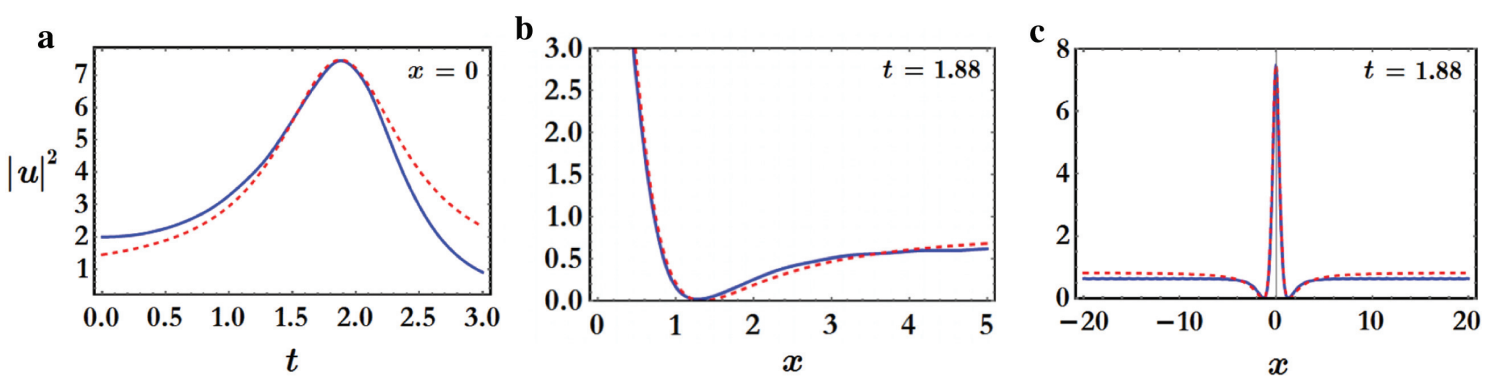

Figure 9: (Colour Online) Dynamics of the initial condition (3) with $\alpha=\sqrt{2}$ and $\beta=1$, for the damped and forced NLS (13). Parameters: $\nu=2, \sigma=1, \gamma=0.02, \Gamma=0.5, \Omega=1, L=100$. (a) Evolution of the density of the center, $|u(0, t)|^{2}$, against the evolution of the density of the center of the PRW (5), $u_{\mathrm{PS}}(x, t-1.88 ; 0.83)$, with $K_{0}=1.55$ and $\Lambda=1.20$. (b) A detail of the spatial profile of the maximum event at $t^{*}=1.88$, close to the right of the two symmetric minima of the exact PRW $u_{\mathrm{PS}}(x, t-1.88 ; 0.83)$. (c) Another view of the numerical density, at time $t^{*}=1.88$, where the extreme event attains its maximum amplitude, for $x \in[-20,20]$. 

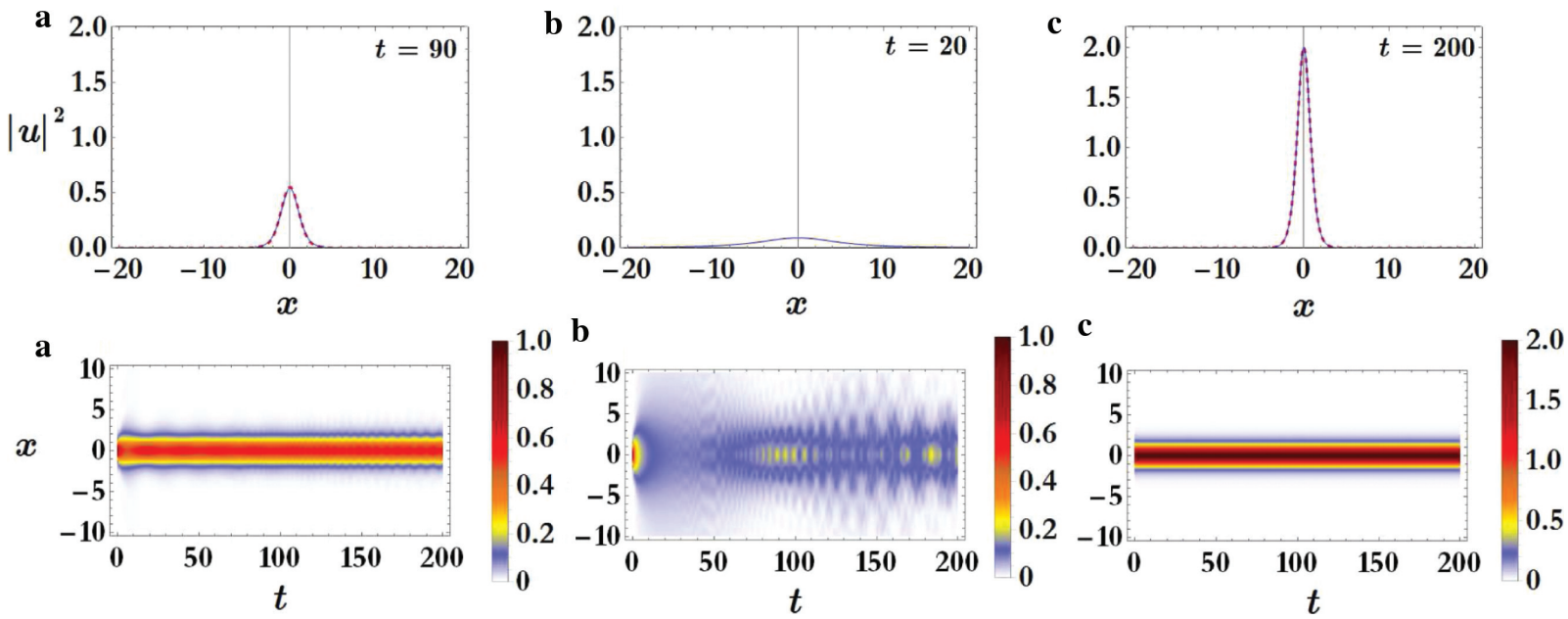

Figure 10: (Colour online) Left column (a): Parameters in (1), $\nu=\sigma=1, \Gamma=0$. The top panel (a) shows a snapshot of the numerical density of the solution with initial condition (2), at $t=90$ [continuous (blue) curve], against the density of an exact stationary soliton $|\Phi|^{2} \approx 0.54 \operatorname{sech}^{2}(0.73 x)$ [dashed (red) curve]. The bottom panel (a) shows a contour plot of the spatiotemporal evolution of the density, for parameters and initial condition as in the top panel (a). Middle column (b): Parameters in (1), $\nu=2, \sigma=1, \Gamma=0$. The top panel (b) shows a snapshot of the density of the numerical solution for the initial condition (2), at $t=20$. The bottom panel (b) shows a contour plot of the spatiotemporal evolution of the density, for parameters and initial condition as in the top panel (b). Right column (c): Parameters in (1), $\nu=2, \sigma=1, \Gamma=0$. The top panel (c) shows a snapshot at $t=200$ of the density of the numerical solution [continuous (blue) curve], with initial condition (3) $(\alpha=\sqrt{2}, \beta=1)$, against the density of the corresponding analytical stationary soliton [dashed (red) curve]. The bottom panel (b) shows a contour plot of the spatiotemporal evolution of the density for parameters and initial condition as in the top panel (c). In all studies, $L=100$.

Setting $2|\theta|=0.54$, we deduce that indeed the numerically detected $|\Phi|^{2}$ corresponds to the density of an exact stationary pulse. The approaching of $|\Phi|^{2}$ is illustrated in the contour plot of the spatiotemporal evolution of the density, shown in the bottom left panel (a). We observe the slight oscillations of the numerical solution around $\Phi$. This oscillatory motion is completely determined by the well-known stability results of [58, Theorem 8.3.1] for standing wave solutions, if applied in the case of the cubic NLS: the requested distance between the initial condition (2) and $\Phi$ is quantitatively significant in the Sobolev norm $H^{1}(\mathbb{R})$, i.e. $\left\|u_{0}-\Phi\right\|^{2}=\int_{-\infty}^{\infty}\left|u_{0}-\Phi\right|^{2} \mathrm{~d} x+$ $\int_{-\infty}^{\infty}\left|\partial_{x} u_{0}-\partial_{x} \Phi\right|^{2} \mathrm{~d} x \approx 0.97$. Due to this distance, the excited numerical solution, although it seems to be almost locked to $\Phi$, exhibits the observed oscillatory behaviour.

The middle column (b) depicts the dynamics of the initial condition (2), for $\nu=2, \sigma=1$. The initial condition disperses, as shown in the snapshot of the density for $t=20$ of the upper panel (b), and the contour plot of its spatiotemporal evolution, shown in the bottom panel (b).

Finally, column (c) depicts the dynamics of the initial condition (3), for $\alpha=\sqrt{2}$ and $\beta=1$, when $\nu=2$ and $\sigma=1$. In this case, the initial condition $u_{0}(x)=$ $\sqrt{2} \operatorname{sech}(x)$ corresponds to the exact stationary pulse (14) at $t=0$, for $\kappa=0, \theta=1$, and the above values of $\nu$ and $\sigma$. The snapshot of the numerical density [(blue) continuous curve], at $t=200$, shown in the upper right panel (c), fits exactly to the density of the exact stationary solution [(red) dashed curve]. The contour plot of the spatiotemporal evolution of the density is an illustration of the wellknown stability of the exact soliton pulse.

It should be also highlighted that for the generalised focusing Hamiltonian NLS

$$
\mathrm{i} u_{t}+\frac{\nu}{2} u_{x x}+\sigma|u|^{2 \delta} u=0, \quad \delta \geq 1,
$$

( $\delta=1$ corresponds to the integrable NLS), rogue waves can be still excited by spatially decaying initial conditions as it was found in [50]. However, the observed extreme waves therein (excited by generic Gaussian wave packets as their width is varied) are decaying to zero. This is a vast difference of the results of [50], with those presented in the present article.

Summarising, comparing the dynamics of the integrable NLS $(\Gamma=0)$ with those of the forced $(\Gamma>0)$ (1) [and damped $(\gamma>0)(13)]$, it is clear that the birth of extreme events for the latter, initiated by vanishing initial conditions, is far from any integrable limit approximation, $[59,60]$, and further justifies the potential existence of thresholds for the driver's amplitude and frequency, with the properties described at the end of Section 2.3. 


\section{Conclusions}

In this work, direct numerical simulations revealed the excitation of Peregrine-type solitonic waveforms, from vanishing initial conditions (possessing an algebraic or exponential spatial decaying rate), for the periodically driven NLS equation. The PRW-type waveforms emerge as first events of the evolution, on the top of a selfinduced finite background. This dynamical behaviour can be understood in terms of the existence and modulation instability of the cw solutions of the model and the preservation of the spatial localisation of the initial condition at the early stages of the evolution. Revisiting the dynamics of the corresponding conservative NLS for the same type of initial conditions, it was shown that the above dynamics should be considered as far from approximations from the integrable limit. We also commented that this behaviour may persist in the linearly damped and forced counterpart, at least under the presence of small damping strengths. Importantly, it appears that the emergence of the Peregrine soliton excited by decaying initial conditions as a universal, coherent structure in the dynamics of the 1D - integrable NLS [61] - as studied therein, in its semiclassical limit scenario $[62,63]$ - can be robust in the presence of forcing and damping. Notably, for the persistence of semiclassical type dynamics in the presence of a spatiotemporally localised driver (pending on the spatial/temporal scales of the latter and the magnitude of the damping strength), we refer to our recent work [64].

Future directions include further investigations on forced and damped NLS models, which may be considered in $1 \mathrm{D}$ and higher dimensional setups, the consideration of various types of forcing (as spatiotemporally localised [64]), the presence of higher order effects, and discrete [65], damped, and forced NLS counterparts. Relevant investigations are in progress and will be considered in future publications.

Acknowledgments: This research is co-financed by Greece and the European Union (European Social Fund - ESF) through the Operational Programme (Human Resources Development, Education and Lifelong Learning 2014-2020) in the context of the project "Localized and quasiperiodic solutions for partial differential equations: Dynamical paths from mathematical ecology to nonlinear physics" (MIS 5004244).

\section{References}

[1] D. H. Peregrine, J. Austral. Math. Soc. B 25, 16 (1983).
[2] E. A. Kuznetsov, Sov. Phys.-Dokl. 22, 507 (1977).

[3] Y. C. Ma, Stud. Appl. Math. 60, 43 (1979).

[4] N. N. Akhmediev, V. M. Eleonskii, and N. E. Kulagin, Theor. Math. Phys. 72, 809 (1987).

[5] E. Pelinovsky and C. Kharif (Eds.), Extreme Ocean Waves, Springer, New York 2008.

[6] C. Kharif, E. Pelinovsky, and A. Slunyaev, Rogue Waves in the Ocean, Springer, New York 2009.

[7] A. R. Osborne, Nonlinear Ocean Waves and the Inverse Scattering Transform, Academic Press, Amsterdam 2010.

[8] M. Onorato, S. Residori, and F. Baronio, Rogue and Shock Waves in Nonlinear Dispersive Media, Springer-Verlag, Heidelberg 2016.

[9] A. Chabchoub, N. P. Hoffmann, and N. Akhmediev, Phys. Rev. Lett. 106, 1 (2011).

[10] A. Chabchoub, N. Hoffmann, M. Onorato, and N. Akhmediev, Phys. Rev. X 2, 1 (2012).

[11] D. R. Solli, C. Ropers, P. Koonath, and B. Jalali, Nature 450, 1054 (2007).

[12] B. Kibler, J. Fatome, C. Finot, G. Millot, F. Dias, et al., Nat. Phys. 6, 790 (2010).

[13] C. Lecaplain, Ph. Grelu, J. M. Soto-Crespo, and N. Akhmediev, Phys. Rev. Lett. 108, 1 (2012).

[14] A. N. Ganshin, V. B. Efimov, G. V. Kolmakov, L. P. MezhovDeglin, and P. V. E. McClintock, Phys. Rev. Lett. 101, 1 (2008).

[15] H. Bailung, S. K. Sharma, and Y. Nakamura, Phys. Rev. Lett. 107, 1 (2011).

[16] W. Cousins and T. Sapsis, Phys. Rev. E 91, 1 (2015).

[17] W. Cousins and T. Sapsis, J. Fluid Mech. 790, 368 (2016).

[18] R. Hirota, J. Math. Phys. 14, 805 (1973).

[19] K. B. Dysthe and K. Trulsen, Phys. Scr. T82, 48 (1999).

[20] A. Ankiewicz, N. Devine, and N. Akhmediev, Phys. Lett. A 373 , 3997 (2009).

[21] A. Calini and C. M. Schober, in: Extreme Ocean Waves (Eds. E. Pelinovsky and C. Kharif), Springer, New York 2008, p. 31.

[22] Y. Wang, L. Song, L. LI, and B. A. Malomed, J. Opt. Soc. Am. B 32, 2257 (2015).

[23] L. H. Wang, K. Porsezian, and J. S. He, Phys. Rev. E 87, 053202 (2013).

[24] A. Ankiewicz, Y. Wang, S. Wabnitz, and N. Akhmediev, Phys. Rev. E 89, 012907 (2014).

[25] Y. Yang, Z. Yan, and B. A. Malomed, Chaos 25, 103112 (2015).

[26] Y. V. Bludov, R. Driben, V. V. Konotop, and B. A. Malomed, J. Opt. 15, 064010 (2013).

[27] H. N. Chan, B. A. Malomed, K. W. Chow, and E. Ding, Phys. Rev. E 93, 012217 (2016).

[28] W. P. Zhong, M. Belic', and B. A. Malomed, Phys. Rev. E 92, 053201, 1 (2015).

[29] J. Cuevas Maraver, P. G. Kevrekidis, D. J. Frantzeskakis, N. I. Karachalios, M. Haragus, et al., Phys. Rev. E 96, 012202 (2017).

[30] J. Cuevas-Maraver, B. A. Malomed, P. G. Kevrekidis, and D. J. Frantzeskakis, Phys. Lett. A 382, 968 (2018).

[31] A. Slunyaev, A. Sergeeva, and E. Pelinovsky, Phys. D 303, 18 (2015).

[32] C. Kharif and J. Touboul, Eur. Phys. J. Special Topics 185, 159 (2010). 
[33] C. Kharif, R. A. Kraenkel, M. A. Manna, and R. Thomas, J. Fluid Mech. 664, 138 (2010).

[34] Y. Kivshar and B. Malomed, Rev. Mod. Phys. 61, 763 (1989).

[35] Z. A. Anastassi, G. Fotopoulos, D. J. Frantzeskakis, T. P. Horikis, N. I. Karachalios, et al., Phys. D 355, 24 (2017).

[36] G. Biondini and D. Mantzavinos, Phys. Rev. Lett. 116, 043902 (2016).

[37] G. Yang, L. Li, and S. Jia, Phys. Rev. E 85, 046608 (2012).

[38] G. Yang, Y. Wang, Z. Qin, B. A. Malomed, D. Mihalache, et al., Phys. Rev. E 90, 062909 (2014).

[39] L. Friedland and A. G. Shagalov, Phys. Rev. E 71, 036206 (2005).

[40] E. Shlizerman and V. Rom-Kedar, Phys. Rev. Lett. 96, 024104 (2006).

[41] E. Shlizerman and V. Rom-Kedar, Phys. Rev. Lett. 102, 03390 (2009).

[42] K. Nozaki and N. Bekki, Phys. Rev. Lett. 50, 1226 (1983).

[43] K. Nozaki and N. Bekki, Phys. Lett. A 102, 383 (1984).

[44] K. Nozaki and N. Bekki, Phys. D 21, 381 (1986).

[45] Y. Li and D. W. McLaughlin, Comm. Math. Phys. 162, 175 (1994).

[46] G. Haller and S. Wiggins, Physica D 85, 311 (1995).

[47] D. Cai, D. W. McLaughlin, and J. Shatah, Phys. Lett. A 253, 280 (1999).

[48] D. Cai, D. W. McLaughlin and K. T. R. McLaughlin, The Nonlinear Schrödinger Equation as Both a PDE and a Dynamical System, Handbook of dynamical systems, vol. 2, North-Holland, Amsterdam 2002, p. 599.

[49] E. Shlizerman and V. Rom-Kedar, Chaos 15, 013107, 1 (2005).

[50] E. G. Charalampidis, J. Cuevas-Maraver, D. J. Frantzeskakis, and P. G. Kevrekidis, Rom. Rep. Phys. 70, 504 (2018).
[51] J. Fujioka, E. Cortés, R. Pérez-Pascual, R. F. Rodrguez, A. Espinosa, et al., Chaos 21, 033120 (2011).

[52] S. Randoux, P. Suret, A. Chabchoub, B. Kibler, and G. El, Phys. Rev. E 98, 022219 (2018).

[53] O. Kimmoun, H. C. Hsu, B. Kibler, and A. Chabchoub, Phys. Rev. E 96, 022219 (2017).

[54] S. Randoux, P. Suret, and Gennady, Sci. Rep. 6, 29238 (2016).

[55] J. M. Ghidaglia, Ann. Inst. Henri Poincaré 5, 365 (1988).

[56] O. Goubet, Appl. Anal. 60, 99 (1996).

[57] D. Cai, A. R. Bishop, N. G.-Jensen, and B. A. Malomed, Phys. Rev. E 49, R1000 (R) (1994).

[58] T. Cazenave, Semilinear Schrödinger Equations, Courant Lecture Notes 10 (American Mathematical Society, 2003).

[59] M. Onorato and D. Proment, Phys. Lett. A 376, 3057 (2012).

[60] M. Brunetti, N. Marchiando, N. Berti, and J. Kasparian, Phys. Lett. A 378, 1025 (2014).

[61] A. Tikan, C. Billet, G. El, A. Tovbis, M. Bertola, et al., Phys. Rev. Lett. 119, 033901 (2017).

[62] M. Bertola and A. Tovbis, Comm. Pure Appl. Math Comm. Pure Appl. Math. 66, 678 (2009).

[63] R. H. J. Grimshaw and A. Tovbis, Proc. R. Soc. A 469, 20130094 (2013).

[64] G. Fotopoulos, D. J. Frantzeskakis, N. I. Karachalios, P. G. Kevrekidis, V. Koukouloyannis, et al., Extreme Wave Events for a Nonlinear Schrödinger Equation with Linear Damping and Gaussian Driving, https://arxiv.org/abs/1812.05439.

[65] P. G. Kevrekidis, The Discrete Nonlinear Schrödinger Equation: Mathematical Analysis, Numerical Computations and Physical Perspectives, Springer-Verlag, Berlin, Heidelberg 2009. 